\title{
Tools for Feebly Interacting Particles
}

\author{
Luc Darmé ${ }^{a, *}$ \\ ${ }^{a}$ Istituto Nazionale di Fisica Nucleare, Laboratori Nazionali di Frascati, \\ C.P. 13, 00044 Frascati, Italy
}

E-mail: luc.darme@lnf.infn.it

Feebly interacting particles (FIPs) in the $\mathrm{MeV}$ to $\mathrm{GeV}$ range can be studied from a large variety of past, current and future experiments. While the number of phenomenological and experimental studies on FIPs has dramatically increased in recent years, the available choice of tools has remained limited. We will briefly review existing tools and present some of the current challenges, focusing mainly on so-called beam dump experiments. We present in particular the recasting code "DarkEFT" along with a database of relevant standard model data for FIPs production in proton beam dump experiments.

Tools for High Energy Physics and Cosmology - TOOLS2020

2-6 November, 2020

Institut de Physique des 2 Infinis (IP2I), Lyon, France

\footnotetext{
${ }^{*}$ Speaker
} 


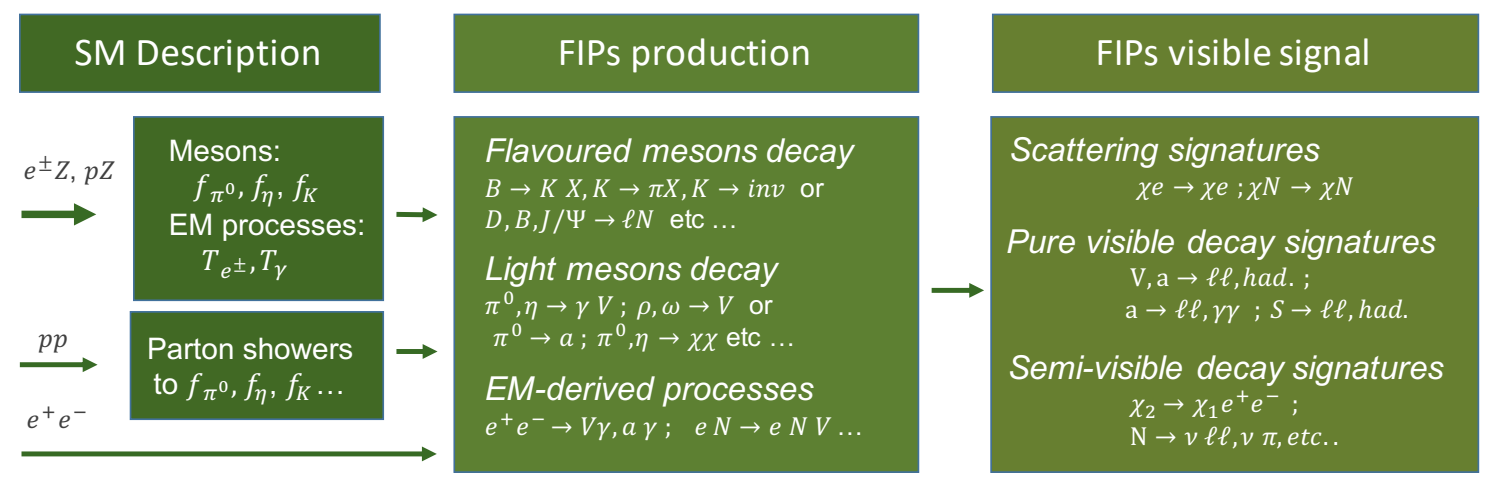

Figure 1: Schematic simulation chain for beam dump experiments. Typical relevant processes are shown for a dark Higgs $S$, a dark photon $V$, an axion-like particle $a$, dark fermions $\chi_{i}$ and a heavy neutral lepton $N$. We notes $f_{M}(\theta, E)$ the meson differential distributions and $T_{e / \gamma}(E)$ the track lengths (see the main text).

\section{Introduction}

Feebly Interacting Particles (FIPs) are currently the subject of an intense experimental program spanning both astrophysical searches and laboratory-based experiments. FIPs can be loosely defined as new (quasi-) ${ }^{1}$ neutral particles which interact with the Standard Model (SM) via suppressed new interactions. They can appear in various new physics scenarios aiming at providing a dark matter candidate, generating neutrino masses, solving the strong CP problem, etc... Interestingly, FIPs can only interact with the SM in a limited number of ways, which can be loosely classified via the dimension of the "portal" operator mediating their interaction with SM fields. Indeed, since FIPs are SM gauge singlet, they must be associated with operators similarly singlet. Not many such operators can be written purely in term of SM fields, thus leading to the "portal operator" classification. Up to dimension 3, one can list: $O_{2}^{H} \equiv H H^{\dagger}$, the Higgs portal, $O_{2}^{V}=F^{\mu v}$ the vector portal based on the hypercharge field strength , $O_{5 / 2} \equiv \bar{L} H$ the neutrino portal at dimension $5 / 2$, with $L$ a left-handed lepton doublets, and the fermion portal $O_{3} \equiv \bar{\psi} \Gamma \psi$ with $\psi$ any SM fermions and $\Gamma$ a combination of gamma matrices. ${ }^{2}$ It is clear that while these operators are gauge singlets, they are not Lorentz invariants, hence the possible FIPs interaction are also constrained to adopt a particular Lorentz structure. Altogether, most of the phenomenology of any FIPs model will be derived from its portal operator, as it dictates its production rates and a large part of its possible detection mechanisms.

In the following we will review the status of numerical and analytical tools currently available to study the production and possible detection of FIPs in the $\mathrm{MeV}$ to $\mathrm{GeV}$ range. We focus on laboratory-based experiments, thus setting aside the flourishing area of light dark matter searches, and will mostly consider beam dump-based experiments, as these are the most challenging to simulate. The complete simulation chain, shown in Fig. 1 is typically separated in three stages: SM description, FIPs production and FIPs visible signatures. We will consider each stage in turn in Sec. 2 and Sec. 3, before briefly presenting recasting tools in Sec. 4.

\footnotetext{
${ }^{1}$ Milli-charged particle are typically included as FIP candidate, while having by definition a tiny electromagnetic charge.

${ }^{2}$ Note that the derivative term $H \partial_{\mu} H$ provides another dimension 3 portal.
} 


\section{Standard Model simulation}

In order to simulate FIPs production in beam dump experiment, we need first an accurate description of the particle showers occurring in the target material. This implies both extracting (flavoured) mesons and leptons/photon distributions. In almost all relevant cases, the typical dimension of the hadronic (electromagnetic) shower in the beam dump target is negligible with respect to the distance with the detector apparatus. Therefore, one can integrate over the entire shower, loosing the precise meson production point, but dealing directly with integrated distributions function only of the energy and on the polar angle with respect to the beam direction $\theta$.

Mesons-induced processes The relevant quantities are the energy and angular distributions of mesons: $f_{M}(\theta, E)$. Depending on the experiment, one typically needs to find the above distribution of mesons, created either from a $p p$ collision (LHC-based long-lived particle experiments) or from a $p N$ interaction (where $N$ is a nucleus in proton beam dump). Although closed analytical form does not exist, several empirical distributions are available, based on the results from past beam dump experiments. These include for instance (see [1]):

- BMTP (Bonesini, Marchionni, Pietropaolo, Tabarelli de Fatis) [2], which is based on various experiments using the high-energy beam at SPS (typically with a Beryllium target). It can be used to model the distributions of light unflavored mesons for the higher energy beam dumps, and also includes Kaons distributions.

- Sanford-Wang [3]. This generic parametrisation has been adapted to various cases, and in particular to the Booster Neutrino Beam facility with a $8 \mathrm{GeV}$ proton beam impinging on a thin Beryllium target [4]. ${ }^{3}$ This distribution can also be used for Kaons.

- Burman-Smith [5]. Used for the low-energy $0.8 \mathrm{GeV}$ proton beam at the LSND experiments at LAMPF. ${ }^{4}$

Although the above distributions are particularly efficient in obtaining a fast estimate of the distributions, they typically tend to underestimate the secondary production of light mesons which typically arise in hadronic showers. As an example, we show in Fig. 2 the distribution of $\pi^{0}$ mesons obtained via both the empirical distributions described above, and the full results based on a GEANT4 simulation as available from [16], for the case of a MiniBooNE-type experiment, and for a DUNE-type experiment. It is clear that both empirical distributions fail to account properly from the multiplicity of low-energy mesons, with in particular the Sanford-Wang parametrisation proving a poor fit to the actual distribution (this was expected since it was designed to describe the production in a thin Be target, while the off-target configuration of MiniBooNE involves instead mostly the iron beam dump).

When only the primary interaction is relevant, or when the distributions of heavier mesons mesons (for instance $B$-mesons) are needed, one can use standard high energy numerical tools such

\footnotetext{
${ }^{3}$ Note that the same distribution has also been used in [1] to find the mesons distributions produced in MiniBooNE off-target configuration.

${ }^{4}$ For LSND, one needs to weight the distribution to account for the two different experimental stages: water then high-Z metal [6]
} 
as Pythia 8 [7], or other tools such as the ones included in the CRMC package: 5 EPOS@LHC [8], QGS JETII [9], etc ... Finally, when the precise interaction point may be relevant (long-lived FIPs produced from atmospheric showers for instance) or when the complete description of secondary productions matter, one needs to rely on full-fledged MC tools, such as GEANT4 [10] or FLUKA [11].

Lepton/photon induced processes As for mesons, in most cases, only the angular and energy distributions of the produced FIPs will be relevant. However, since leptons and photons typically produce FIPs via non-SM interactions with the target material, one needs to have access to the "total length traveled in the target for each particle with a given angle and energy". This information is enclosed in the "differential track length" $\partial T_{e / \gamma}(E)$, which can be roughly understood as the effective target length seen by photon/lepton of a given angle and energy. As the electromagnetic shower develops, the energy from the primary particle is transformed into large number of low energy photons and electrons/positrons. Thus, differential track lengths tend to strongly increases at low energies.

For pure electromagnetic showers, reliable analytical tools have been developed long ago to describe the track lengths distribution (see for instance [12]), including the cut-off effects from the increasing importance of ionisation events for the low-energy tails (see, e.g. [13, 14]). For instance following Tsai [12] in the case of a primary electron beam, one has:

$$
\partial T_{e / \gamma}(E)=\int_{0}^{t_{\mathrm{tar}}} d t I_{\gamma, e}(t, E) \quad \text { where }\left\{\begin{array}{l}
I_{\gamma}(t, E)=\frac{1}{E} \frac{\left(1-E / E_{0}\right)^{4 t / 3}-e^{-7 t / 9}}{\frac{7}{9}+\frac{4}{3} \ln \left(1-E / E_{0}\right)} \\
I_{e}(t, E)=\frac{1}{E} \frac{\left.\left[\ln \left(E_{0} / E\right)\right)\right]^{4 t / 3-1}}{\Gamma(4 t / 3)}
\end{array},\right.
$$

where $t_{\text {tar }}$ is the target length in unit of radiation length. Those analytical descriptions are typically reliable as long as the target is not too thin, so that numerous bremsstrahlung/pair-production events can occur and subsequently develop the electromagnetic shower. Note that these descriptions do not include the angular distribution in the shower.

Database approach Since the SM part of an experiment has to be simulated only once and that the required information to simulate the FIPs production processes can be included in two dimensional functions, it is clear that a database approach can be used. For each experimental setup (for instance, a proton beam dump at a given energy), one can generate once-and-for-all the required track lengths and mesons distributions information. This approach was used in [15] and put to practice in releasing a database the Zenodo online repository [16] which covers a large range of existing proton beam dump experiments. We list the experimental setups currently available in Table 1. A more direct interface with the code MadDump [17] is currently under work. This will allow the user to directly download the required distribution from this $\mathrm{MC}$ tool to generate the differential production rate for any given FIPs model. Fig. 2 provides an illustration of the possible use of this database, with the GEANT4 data being directly extracted from [16].

\footnotetext{
${ }^{5}$ Available at this url: https://web.ikp.kit.edu/rulrich/crmc.html.
} 


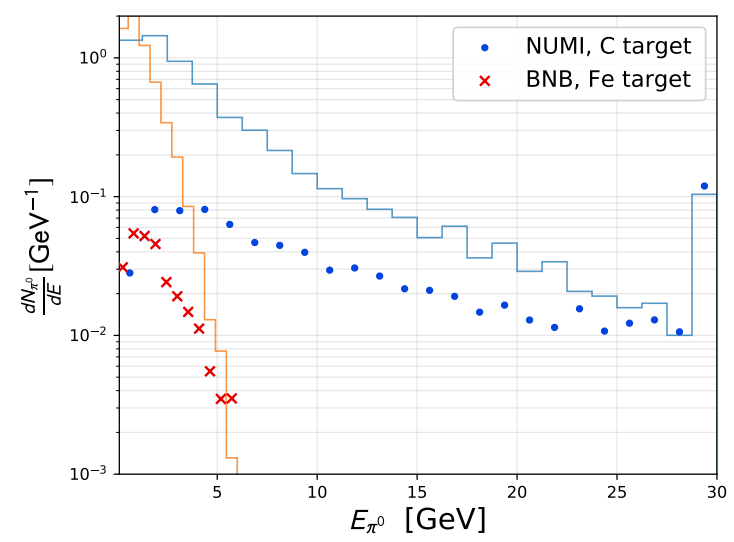

Figure 2: Comparison of the differential $\pi^{0}$ yield per proton on target from a $120 \mathrm{GeV}$ proton impinging on a thick graphite target (NUMI, blue) and for a $8 \mathrm{GeV}$ proton beam on an iron target (BNB, red). The histograms represent the full result from a GEANT4 simulation as described in $[15,16]$, while the points are the result for a BMTP and Sanford-Wang distribution respectively. The selections described in Table 1 are applied. The distributions are normalised to $1 \pi^{0}$ per proton on target for the BMTP and Sanford-Wang distributions, and $6.2(0.25) \pi^{0}$ per proton on target for the full GEANT4 results for the NuMI (resp. BNB) case. The last bin includes the overhead.

\begin{tabular}{|c|c|c|c|c|c|}
\hline Experiment & $E_{\text {beam }}^{\text {kin }}$ & Target & $E_{\text {cut }}, \theta_{\text {cut }}$ & $N_{\pi^{0}}$ & $T_{e^{+} \text {,tot }}(\mathrm{cm})$ \\
\hline \hline MiniBooNE [18] & $8 \mathrm{GeV}$ & Steel & $E>10 \mathrm{MeV}, \sin \theta_{\text {cut }}<0.2$ & 0.25 & 2.8 \\
NO $v$ A [18] & $120 \mathrm{GeV}$ & $\mathrm{C}$ & $E>50 \mathrm{MeV}, \sin \theta_{\text {cut }}<0.2$ & 3.8 & 35 \\
DUNE [19] & $120 \mathrm{GeV}$ & $\mathrm{C}$ & $E>50 \mathrm{MeV}, \sin \theta_{\text {cut }}<0.2$ & 6.2 & 396 \\
SHiP [20] & $400 \mathrm{GeV}$ & Mostly Mo & $E>100 \mathrm{MeV}, \sin \theta_{\text {cut }}<0.2$ & 23 & 240 \\
\hline
\end{tabular}

Table 1: Experiments currently included with the database [16]. All tracks are assumed to satisfy the cuts $E_{\text {cut }} / \theta_{\text {cut }}$ shown in Table. We show the $\pi^{0}$ multiplicity after the cuts, as well as the total track length for positrons of all energy.

\section{FIPs production and decay}

New physics search for FIPs can be either "indirect", looking for missing energy signatures for instance, or "direct", producing FIPs then searching for scattering or decay signals in a detector. The former relies mostly on producing FIPs in the first place and therefore depends primarily on the portal interactions. The latter is on the other hand much more sensitive to the details on the FIPs model, and in particular to the possible presence of an accompanying dark sector.

Production One of the dominant mechanism of FIPs production originates from non-SM decay of mesons. Although conceptually simple, quantitative predictions can be challenging to obtain since predicting accurately the mesons decay rates in the SM is still an active area of research. Currently no automatic tool is available, even for the heavier mesons, primarily due to the fact that the presence of new light states prevents the use of standard Effective Field Theory tools. On the other hand, many of the relevant decays have been estimated throughout the years using various techniques, such as chiral perturbation theory (see e.g. [21]) or Vector Meson Dominance (see 
e.g. [22]) and can be often adapted to the precise case at hand. When the full differential rates are relevant, they can be usually obtained by combining the above analytical insight with mesons distributions obtained following the procedure described in the previous sections.

For lepton and photon-based production process

es, one needs to use instead the track-length formalism. The number of FIPs produced via a process with cross-section $\sigma(E)$ is given by:

$$
\mathcal{N}=\frac{\mathcal{N}_{A} X_{0} \rho}{A} \int_{0}^{E_{i n i}} d E \frac{d T_{ \pm}(E)}{d E} \sigma(E)
$$

with $X_{0}$ is the radiation length of the material, $A$ its atomic mass with $\mathcal{N}_{A}=6.022 \times 10^{23}$, and $\rho$ its mass density. If the angular distribution is needed, one then needs to consider instead differential cross-section and use $\frac{\partial T}{\partial E \partial \theta}$. Numerically, this can be done either manually (by simulating events for different incident particle energy $E$, then combining them by weighting by the track length), or automatically using MADDumP. ${ }^{6}$ An important particular case is FIPs production via interactions with the electromagnetic field sourced by a nucleus. The interaction needs to include form factors accounting for the screening from the electrons in the atomic cloud and from the finite nuclear size (see e.g. [23]). ${ }^{7}$ Some limitations of this approach in the case of axion-like particle FIP are presented in [24]. Finally, we note that various other MC generators can be used to simulate the FIPs production in $e^{+} / e^{-}$processes, such as BABAYAGA @NLO [25] or CALCHEP [26].

Importantly, this production stage is often enough to obtain constraints on FIPs based on missing energy searches (such as the recent NA64 results [27]). However, a large class of experiments further try to measure visible FIPs signatures, requiring yet another layer of simulations, as described in the next section.

Detection Although direct detection techniques of "visible" FIP signatures can lead to much stronger bounds than "indirect" searches, they suffer from a large dependence to the details of the FIP model and of the possible presence of an accompanying dark sector. Typical searches rely either on scattering signatures (as for light dark matter scenarios) or from (semi)-visible decays (as for heavy neutral leptons decay for instance). Many private codes exist for the different setups studied in the literature. The main public codes are

- BDNMC [1]. Build in $\mathrm{C}++$, this code is designed mainly for proton beam dump experiments. It includes various empirical distribution for mesons as listed above, as well as various hardcoded dark photon production processes. It can simulate out of the box scattering signatures for light dark matter via a vector portal in most proton beam dump experiments. Interestingly, it can be easily modified to include decay signatures and various experimental cuts.

- MadDump [17]. MadDump is a plugin of MadGraph5_aMC@NLO [28] designed to assist the simulation of events in various experimental setups by simulating both the production and detection, in possibly separated locations. Owing to its integration within the MADGRAPH5_aMC@NLO environment, it can be used on any UFO model files and can thus accommodate various FIPs model. Mesons distributions needs to generated independently then loaded. It can simulate scattering signals and has a simple displacement decay mode.

${ }^{6}$ The relevant version is still in development at the time of writing but can be obtained directly from the authors.

${ }^{7}$ Both can be directly integrated within the MADGraPH5_aMC@NLO framework, see e.g. [15]. 


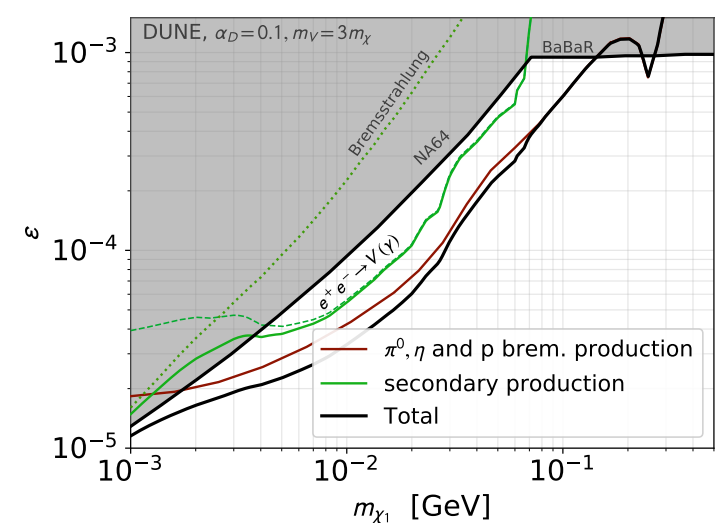

(a)

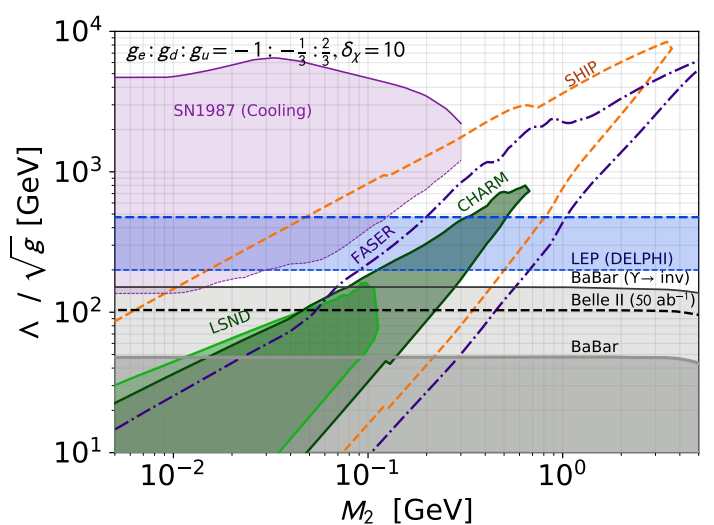

(b)

Figure 3: (a) Projected reach of the DUNE near detector for a light dark matter model with vector portal, shown in term of the kinetic mixing $\varepsilon$, extracted from [15]. The grey region represents the exclusion bounds from the BaBar [30] and NA64 [27] collaborations. The rust line represents our estimate based on hadronic processes only, the solid green line our estimate based on secondary production only, and the thick black line is the combination of both. (b) Limits and projected sensitivity to the vector operator effective scale $\Lambda / \sqrt{g}$ in the case of effective coupling electromagnetically-aligned as function of the dark fermion mass $M_{2} \gg M_{1}$, extracted from [31]. The grey region indicates coverage from mono-photon at BaBar [32], dashed grey line Belle-II projections [32]. The shaded blue region is the mono- $\gamma$ limit from LEP [33]. Limits from $\chi_{2} \rightarrow \chi_{1} e^{+} e^{-}$: the green (dark green) regions are the exclusion from LSND [6] (CHARM [34]). We show a projection for FASER [35] in dashed purple and SeaQuest in red (Phase-II defined in [36]). The 10 events reach by SHiP is shown in dashed orange. The purple region represents the limit from cooling of SN1987A [37]. The normalised splitting is defined as $\delta_{\chi}=\left(M_{2}-M_{1}\right) / M_{1}$.

- Alpaca [24]. A Fortran-based code designed primarily for long-lived axion-like particle production via coherent proton-(electron-)nucleus interaction and their subsequent di-photon decays.

Note that starting from version 1.9. the analysis tool MADANALYsis 5 [29] also includes various tools to study long-lived particles, providing an additional mean to deal with the output of the above MC generators.

We show in Fig. 3 an example of the result from a complete simulation chain, with a projected reach for the future DUNE detector (extracted from [15]). The production rates, including lepton and meson induced processes are obtained via MADDuMP, using track lengths and mesons distribution extracted from a full GEANT4 simulation of the showers in the target and packaged in the public database [16]. The final number of scattering events is simulated with BDNMC, with the experimental cut directly included at the MC truth level.

\section{Recasting approach}

An interesting third category of tools rely on the fact that since most of the FIP phenomenology depends on the nature of the portal operator, there is a natural recasting path to re-interpret 
experimental searches between FIPs model sharing the same portal operator. Currently, two portals have been included in public codes, both are python-based.

- DarkCast [38]. It can be used to re-interpret vanilla dark photon searches in term of a generic new vector boson and therefore covers most cases of FIPs with vector portal. Note that only the signatures from the dark photon itself are included (including long-lived decay searches)

- DARKEFT [31]. Used to re-interpret various limits in terms of fermion portal four-fermions operators:

$$
\frac{1}{\Lambda^{2}}\left(\bar{\chi}_{2} \Gamma \chi_{1}\right) O_{3},
$$

where $O_{3}$ is a fermion portal operator and $\Gamma$ a combination of gamma matrices, and can be therefore used as the heavy mediator limits of various other portals. It includes the possibility to recast existing inelastic dark matter limits in beam dump experiments to constrain the case with two fermionic FIPs $\chi_{2}, \chi_{1}$ with the decay channel $\chi_{2} \rightarrow \chi_{1} e^{+} e^{-}$.

In both cases, most limits are derived in an analytic fashion by estimating first the most generic production and mesons decay rates for the portal at hand. The prediction for user-chosen couplings is then compared with the rates used in the experimental analysis to obtain a recasted limit. When dealing with long-lived FIPs decay constraints, the re-interpretation process is more involved. Taking the example of the inelastic dark matter recast implemented in DARKEFT, the final expected number of events in the beam dump experiment can be expressed as:

$$
\mathcal{N} \simeq \mathcal{N}_{\text {prod }} \times \mathcal{E} \times \mathcal{P}_{\text {sig }},
$$

where $\mathcal{N}_{\text {prod }}$ is the production rate, $\mathcal{E}$ is the experimental efficiency and $\mathcal{P}_{\text {sig }}$ is the decay probability. One first typically assumes that they are mostly a function of the mass of the FIP, so that they can be factored out when determining the new limits as function of the mass. For a beam dump experiment of length $L$ at a distance $D$ from the beam target, one can then follows a three steps process:

1. The typical number of produced dark sector particles is estimated, both for the existing search, $\mathcal{N}_{\text {prod }}^{\mathrm{DP}}$ and for the new theory $\mathcal{N}_{\text {prod }}^{\text {new }}$.

2. For the very long-lived case, where $c \tau \gamma \gg D, L$ (with $\gamma$ the FIP boost factor), the geometric parameter of the experiments cancels out of the ratio

$$
\frac{\mathcal{P}_{\text {sig }}^{\mathrm{DP}}}{\mathcal{P}_{\text {sig }}^{\text {new }}}=\frac{\Gamma_{\mathrm{DP}}}{\Gamma_{\text {new }}},
$$

where the decay rates $\Gamma_{\mathrm{DP}}$ and $\Gamma_{\text {new }}$ are then estimated analytically in both cases, and the coupling dependence extracted to find the new limits.

3. For the short-lived case (corresponding typically to the upper limit, when the FIP decays before reaching the detector), the decay probability takes an exponential form

$$
\exp \left(-\frac{(L+D) \Gamma_{\mathrm{DP}}}{c \hbar \gamma}\right) \mathcal{N}_{\text {prod }}^{\mathrm{DP}}=\exp \left(-\frac{(L+D) \Gamma_{\text {new }}}{c \hbar \gamma}\right) \mathcal{N}_{\text {prod }}^{\text {new }} .
$$


which is then solved to obtain the new limits. Note that the average FIP boost factor plays an important role and should be carefully estimated for each recasted experimental search. ${ }^{8}$

We show in Fig. 3 an example of limits obtained for a fermion portal setup based on DARKEFT (in the case of an inelastic dark matter setups with two dark fermions $\chi_{2}$ and $\chi_{1}$, interacting with the SM via four-fermion effective operators which also mediates the decay $\chi_{2} \rightarrow \chi_{1} e^{+} e^{-}$). The plot combines limits from mono-photon searches, long-lived FIP decay in beam dumps, rare meson decay and astrophysical constraints.

\section{Conclusion}

We have shown in this proceeding that signatures of FIPs in beam dump experiments, and more generally in intensity frontier experiments can be typically simulated in three stages. First, the simulation of the SM part, which is computationally intensive but can then be treated in a once-and-for-all approach, as we have illustrated by presenting a database for various proton beam experiments. Second, the estimation of the FIPs production rates, which are mostly of function of the portal operator used to couple the FIP to the SM, thus offering a systematic way of classification. Finally the simulation of FIPs "visible" signatures, which is both more model dependent and computationally intensive, requiring additional developments of dedicated MC tools. The possibility of relying on the portal operator formalism to offer fast re-interpretation of existing experimental search to any FIPs scenario was briefly described.

Altogether, and although several interesting developments have been put forward in recent years, it is clear that much remain to be done to attain the level of precision available at the highenergy frontier. Since FIPs are currently the target of a flourishing experimental effort, this issue will become more pressing in the coming years.

\section{References}

[1] P. deNiverville, C. Y. Chen, M. Pospelov and A. Ritz, Phys. Rev. D 95 (2017) no.3, 035006 doi:10.1103/PhysRevD.95.035006 [arXiv:1609.01770 [hep-ph]].

[2] M. Bonesini, A. Marchionni, F. Pietropaolo and T. Tabarelli de Fatis, Eur. Phys. J. C 20 (2001), 13-27 doi:10.1007/s100520100656 [arXiv:hep-ph/0101163 [hep-ph]].

[3] J. R. Sanford and C. L. Wang, PRINT-67-1023.

[4] A. A. Aguilar-Arevalo et al. [MiniBooNE], Phys. Rev. D 79 (2009), 072002 doi:10.1103/PhysRevD.79.072002 [arXiv:0806.1449 [hep-ex]].

[5] R. L. Burman and E. S. Smith, LA-11502-MS.

[6] L. Darmé, S. Rao and L. Roszkowski, JHEP 12 (2018), 014 doi:10.1007/JHEP12(2018)014 [arXiv: 1807.10314 [hep-ph]].

\footnotetext{
${ }^{8} \mathrm{An}$ important comment in this respect is that in the short-lived case, FIPs production rates are typically very large at the beam dump. The limit is then determined by the subset of FIPs produced with the largest possible boost factor.
} 
[7] T. Sjöstrand, S. Ask, J. R. Christiansen, R. Corke, N. Desai, P. Ilten, S. Mrenna, S. Prestel, C. O. Rasmussen and P. Z. Skands, Comput. Phys. Commun. 191 (2015), 159-177 doi:10.1016/j.cpc.2015.01.024 [arXiv:1410.3012 [hep-ph]].

[8] T. Pierog, I. Karpenko, J. M. Katzy, E. Yatsenko and K. Werner, Phys. Rev. C 92 (2015) no.3, 034906 doi:10.1103/PhysRevC.92.034906 [arXiv:1306.0121 [hep-ph]].

[9] S. Ostapchenko, Phys. Rev. D 83 (2011), 014018 doi:10.1103/PhysRevD.83.014018 [arXiv:1010.1869 [hep-ph]].

[10] J. Allison, J. Apostolakis, S. B. Lee, K. Amako, S. Chauvie, A. Mantero, J. I. Shin, T. Toshito, P. R. Truscott and T. Yamashita, et al. Nucl. Instrum. Meth. A 835 (2016), 186-225 doi:10.1016/j.nima.2016.06.125

[11] T. T. Böhlen, F. Cerutti, M. P. W. Chin, A. Fassò, A. Ferrari, P. G. Ortega, A. Mairani, P. R. Sala, G. Smirnov and V. Vlachoudis, Nucl. Data Sheets 120 (2014), 211-214 doi:10.1016/j.nds.2014.07.049

[12] Y. S. Tsai and V. Whitis, Phys. Rev. 149 (1966), 1248-1257 doi:10.1103/PhysRev.149.1248

[13] B. Rossi and K. Greisen, Rev. Mod. Phys. 13 (1941), 240-309 doi:10.1103/RevModPhys.13.240

[14] P. Lipari, Phys. Rev. D 79 (2009), 063001 doi:10.1103/PhysRevD.79.063001 [arXiv:0809.0190 [astro-ph]].

[15] A. Celentano, L. Darmé, L. Marsicano and E. Nardi, Phys. Rev. D 102 (2020) no.7, 075026 doi:10.1103/PhysRevD.102.075026 [arXiv:2006.09419 [hep-ph]].

[16] Celentano, Andrea, Darmé, Luc, Marsicano, Luca and Nardi, Enrico [Data set]. Zenodo. http://doi.org/10.5281/zenodo.3925273

[17] L. Buonocore, C. Frugiuele, F. Maltoni, O. Mattelaer and F. Tramontano, JHEP 05 (2019), 028 doi:10.1007/JHEP05(2019)028 [arXiv:1812.06771 [hep-ph]].

[18] A. A. Aguilar-Arevalo et al. [MiniBooNE DM], Phys. Rev. D 98 (2018) no.11, 112004 doi:10.1103/PhysRevD.98.112004 [arXiv:1807.06137 [hep-ex]].

[19] V. De Romeri, K. J. Kelly and P. A. N. Machado, Phys. Rev. D 100 (2019) no.9, 095010 doi:10.1103/PhysRevD.100.095010 [arXiv:1903.10505 [hep-ph]].

[20] M. Anelli et al. [SHiP], [arXiv:1504.04956 [physics.ins-det]].

[21] F. Bishara, J. Brod, B. Grinstein and J. Zupan, JCAP 02 (2017), 009 doi:10.1088/14757516/2017/02/009 [arXiv:1611.00368 [hep-ph]].

[22] T. Fujiwara, T. Kugo, H. Terao, S. Uehara and K. Yamawaki, Prog. Theor. Phys. 73 (1985), 926 doi:10.1143/PTP.73.926 
[23] K. J. Kim and Y. S. Tsai, Phys. Rev. D 8 (1973), 3109 doi:10.1103/PhysRevD.8.3109

[24] L. Harland-Lang, J. Jaeckel and M. Spannowsky, Phys. Lett. B 793 (2019), 281-289 doi:10.1016/j.physletb.2019.04.045 [arXiv:1902.04878 [hep-ph]].

[25] L. Barze, G. Balossini, C. Bignamini, C. M. C. Calame, G. Montagna, O. Nicrosini and F. Piccinini, Eur. Phys. J. C 71 (2011), 1680 doi:10.1140/epjc/s10052-011-1680-8 [arXiv:1007.4984 [hep-ph]].

[26] A. Belyaev, N. D. Christensen and A. Pukhov, Comput. Phys. Commun. 184 (2013), 1729-1769 doi:10.1016/j.cpc.2013.01.014 [arXiv:1207.6082 [hep-ph]].

[27] D. Banerjee, V. E. Burtsev, A. G. Chumakov, D. Cooke, P. Crivelli, E. Depero, A. V. Dermenev, S. V. Donskov, R. R. Dusaev and T. Enik, et al. Phys. Rev. Lett. 123 (2019) no.12, 121801 doi:10.1103/PhysRevLett.123.121801 [arXiv:1906.00176 [hep-ex]].

[28] J. Alwall, R. Frederix, S. Frixione, V. Hirschi, F. Maltoni, O. Mattelaer, H. S. Shao, T. Stelzer, P. Torrielli and M. Zaro, JHEP 07 (2014), 079 doi:10.1007/JHEP07(2014)079 [arXiv:1405.0301 [hep-ph]].

[29] E. Conte and B. Fuks, Int. J. Mod. Phys. A 33 (2018) no.28, 1830027 doi:10.1142/S0217751X18300272 [arXiv:1808.00480 [hep-ph]].

[30] J. P. Lees et al. [BaBar], Phys. Rev. Lett. 119 (2017) no.13, 131804 doi:10.1103/PhysRevLett.119.131804 [arXiv:1702.03327 [hep-ex]].

[31] L. Darmé, S. A. R. Ellis and T. You, JHEP 07 (2020), 053 doi:10.1007/JHEP07(2020)053 [arXiv:2001.01490 [hep-ph]].

[32] R. Essig, J. Mardon, M. Papucci, T. Volansky and Y. M. Zhong, JHEP 11 (2013), 167 doi:10.1007/JHEP11(2013)167 [arXiv:1309.5084 [hep-ph]].

[33] P. J. Fox, R. Harnik, J. Kopp and Y. Tsai, Phys. Rev. D 84 (2011), 014028 doi:10.1103/PhysRevD.84.014028 [arXiv:1103.0240 [hep-ph]].

[34] P. deNiverville and C. Frugiuele, Phys. Rev. D 99 (2019) no.5, 051701 doi:10.1103/PhysRevD.99.051701 [arXiv:1807.06501 [hep-ph]].

[35] A. Berlin and F. Kling, Phys. Rev. D 99 (2019) no.1, 015021 doi:10.1103/PhysRevD.99.015021 [arXiv:1810.01879 [hep-ph]].

[36] A. Berlin, S. Gori, P. Schuster and N. Toro, Phys. Rev. D 98 (2018) no.3, 035011 doi:10.1103/PhysRevD.98.035011 [arXiv:1804.00661 [hep-ph]].

[37] W. DeRocco, P. W. Graham, D. Kasen, G. Marques-Tavares and S. Rajendran, Phys. Rev. D 100 (2019) no.7, 075018 doi:10.1103/PhysRevD.100.075018 [arXiv:1905.09284 [hep-ph]].

[38] P. Ilten, Y. Soreq, M. Williams and W. Xue, JHEP 06 (2018), 004 doi:10.1007/JHEP06(2018)004 [arXiv:1801.04847 [hep-ph]]. 